\title{
INDUCED AUTOMORPHISMS OF FREE METABELIAN GROUPS
}

\author{
ORIN CHEIN
}

\begin{abstract}
Let $F$ and $\phi$ respectively be the free group and the free metabelian group of rank $q$. Let $\phi_{n}$ be the $n$th group of the lower central series of $\phi$. We show that, for $q>3$ and for any positive integer $n$, every automorphism of $\phi / \phi_{n}$, which is induced by an automorphism of $\phi$, is induced by an automorphism of $F$. This reopens the question of whether every automorphism of $\phi$ is induced by an automorphism of $F$ if $q>3$.
\end{abstract}

I. Introduction. Bachmuth [2] raised the question of whether every automorphism of a free metabelian group $\phi$ of rank $q$ is induced by an automorphism of the free group $F$ of the same rank $q$. For the case $q=2$, Bachmuth [1, corollary to Theorem 2] showed that the answer is yes. If $q=3$, I proved that the answer is no [3, Theorem 2]. In fact, the mapping

$$
\begin{aligned}
\mu: a & \rightarrow a\left(a,\left(\left(b^{-1}, c^{-1}\right), a^{-1}\right)\right) \\
b & \rightarrow b \\
c & \rightarrow c
\end{aligned}
$$

is an automorphism of $\phi$ which is not induced by an automorphism of $F$. In a certain sense, however, $\mu$ is the only additional automorphism necessary. I.e., for an arbitrary positive integer $n$, every automorphism of $\phi / \phi_{n}$ which is induced by an automorphism of $\phi$ is induced by an automorphism of $\phi$ of the form $\alpha \mu^{\epsilon}$, where $\alpha$ is induced by an automorphism of $F$, and $\epsilon=0$ or 1 [3, p. 629].

In the present paper, we consider the case $q \geqq 4$. While we are not able to determine whether or not every automorphism of $\phi$ is induced by an automorphism of $F$, we prove that the analog of the automorphism $\mu$ no longer plays a significant role. In fact, we prove the following:

THEOREM. If $\phi$ is free metabelian of rank $q \geqq 4$, and if $n$ is any positive integer, then every automorphism of $\phi / \phi_{n}$ which is induced by an automorphism of $\phi$ is induced by an automorphism of the free group $F$ of rank $q$.

Presented to the Society, January 21, 1971; received by the editors November 6, 1970. AMS 1970 subject classifications. Primary 20F55; Secondary 20E05.

Key words and phrases. Free group, free metabelian group, IA automorphism, induced automorphism, lower central series, Bachmuth representation. 
II. Notation and preliminaries. We will denote the free metabelian group of rank $q$ by $\phi$, and the free group of rank $q$ by $F$.

If $G$ is any group, $G_{n}$ will denote the $n$th group of the lower central series of $G$. $G=G_{1} \supseteq G_{2}=G^{\prime} \supseteq G_{3} \supseteq G_{4} \supseteq \cdots$.

The group of automorphisms of $G / G_{n}$ which induce the identity automorphism of $G / G_{m}$ will be denoted by $A\left(G / G_{n} ; G / G_{m}\right)$. The subgroup of $A\left(G / G_{n} ; G / G_{m}\right)$ consisting of those automorphisms which are induced by automorphisms of $G$ will be denoted by $A^{*}\left(G / G_{n} ; G / G_{m}\right)$. And $A^{*}\left(G / G_{n}\right)$ will denote the group of automorphisms of $G / G_{n}$ which are induced by automorphisms of $G$.

The group $A\left(G ; G / G_{2}\right)$ will be called the IA automorphisms of $G$, since they induce the identity automorphism on the abelianized quotient group.

The group of IA automorphisms of $F$ will be denoted by $K$.

If $x_{1}, \cdots, x_{q}$ are are a set of free generators for $F$, then Magnus [4] and Nielsen [5] showed that $K$ is generated by the automorphisms

and

$$
\begin{aligned}
K_{i j}: x_{i} & \rightarrow x_{j} x_{i} x_{j}^{-1} \\
x_{k} & \rightarrow x_{k}, \quad k \neq i,
\end{aligned}
$$

$$
\begin{aligned}
K_{i j k}: x_{i} & \rightarrow x_{i} x_{j} x_{k} x_{j}^{-1} x_{k}^{-1}, & i & \neq k \neq j \neq i, \\
x_{m} & \rightarrow x_{m}, & m & \neq i .
\end{aligned}
$$

To simplify later calculations, we will replace $K_{i j k}$ by

$$
L_{i j k}=K_{i j k} K_{i j} K_{i k} K_{i j}^{-1} K_{i k}^{-1} .
$$

If $\nu$ and $\mu$ are automorphisms, $\nu \mu$ will mean the automorphism resulting when $\nu$ is applied to the set of generators obtained by applying $\mu$ to $x_{1}, \cdots, x_{q}$. Thus

$$
\begin{aligned}
L_{i j k}: x_{i} & \rightarrow x_{j} x_{k} x_{j}^{-1} x_{k}^{-1} x_{i} \\
x_{m} & \rightarrow x_{m} .
\end{aligned}
$$

$K^{*}$ will denote the group of those automorphisms of $\phi$ which are induced by IA automorphisms of $F$.

Since $F / F_{2} \cong \phi / \phi_{2}$, in considering which automorphisms of $\phi$ are induced by automorphisms of $F$, it is enough to restrict ourselves to IA automorphisms. Bachmuth [1] found a faithful matrix representation for the IA automorphisms of $\phi$. (Since the representation is faithful, we will denote an automorphism and its corresponding matrix by the same letter.) The matrices in question are $q$ by $q$ matrices over the ring $Z\left(s_{1}^{ \pm 1}, \cdots, s_{q}^{ \pm 1}\right)$, where $s_{1}, \cdots, s_{q}$ are commuting indeterminates. Substituting $\sigma_{i}$ for $1-s_{i}$ and $1+\sigma_{i}+\sigma_{i}^{2}+\cdots$ for $s_{i}^{-1}$, we can consider the matrices as being over the ring $R$ of power series in the $\sigma_{i}$. 
In this representation, the matrices corresponding to the generators of $K$ are as follows:

$$
\begin{aligned}
L_{123} & =\left(\begin{array}{cccc}
1 & \sigma_{3} & -\sigma_{2} & \\
0 & 1 & 0 & 0 \\
0 & 0 & 1 & \\
& & \\
0 & & I
\end{array}\right), \\
K_{12} & =\left(\begin{array}{ccc}
1-\sigma_{2} & \sigma_{1} & 0 \\
0 & 1 & \\
& & I
\end{array}\right),
\end{aligned}
$$

and the matrix corresponding to any other generator may be found by performing on the rows, columns, and subscripts in one of the above matrices whatever permutation must be performed on the subscripts of $L_{123}$ or $K_{12}$ to obtain the generator in question. For example,

$$
K_{21}=\left(\begin{array}{ccc}
1 & 0 & 0 \\
\sigma_{2} & 1-\sigma_{1} & \\
& & I
\end{array}\right) .
$$

Let $J$ be the ideal of $R$ generated by $\sigma_{1}, \cdots, \sigma_{q}$, and let $J^{n}$ represent the $n$th power of $J$. Then a matrix representing an element in $A\left(\phi ; \phi / \phi_{m}\right)$ will be congruent to the identity matrix $\bmod J^{m-1}([2$, Lemma 4] and $[3, \mathrm{p}$. $608])$. Also, in considering the matrix corresponding to an element of $A\left(\phi \mid \phi_{n} ; \phi / \phi_{m}\right)$, we may disregard terms of degree greater than $n-2$ [3, p. 609].

A matrix $\left(a_{i j}\right)$ represents an IA automorphism of $\phi$ if and only if

(a) det $\left(a_{i j}\right)$ is a unit when converted back into $Z\left(s_{1}^{ \pm 1}, \cdots, s_{q}^{ \pm 1}\right)$,

(b) $\sum_{j=i}^{a} \sigma_{j} a_{i j}=\sigma_{i}[1$, Theorem 1].

If in considering a matrix $M$ as an automorphism in $A^{*}\left(\phi\left|\phi_{n} ; \phi\right| \phi_{m}\right)$ we do disregard terms of degree greater than $n-2$, then condition (b) remains, but condition (a) is replaced by the condition

(c) trace $(M-I)=0$, provided that $n \geqq 4([2$, Lemma 7] and [3, p. 609]). (For $n=3$, we can disregard conditions (a) and (c) entirely.)

If we are interested in matrices corresponding to automorphisms in $A\left(\phi / \phi_{n} ; \phi / \phi_{n-1}\right)$, we need only retain condition (b) [3, p. 609]. Therefore, to determine a matrix corresponding to an automorphism in 
$A\left(\phi \mid \phi_{n} ; \phi / \phi_{n-1}\right)$, it is sufficient to determine those terms in each $a_{i j}$ which involve $\sigma_{k}$ for $k>j$. Since all terms are of degree $n-2$, the number of possible terms for a given $a_{i j}$ is the number of ways we can place $q-1$ spaces among a row of $n-2$ objects $=\left(\begin{array}{c}n+q-3 \\ q-1\end{array}\right)$. The number of terms which involve no $\sigma_{k}$ with $k>j$ is $\left(\begin{array}{c}n+j-3 \\ j-1\end{array}\right)$. Therefore, there are

$$
q \sum_{j=1}^{a}\left\{\left(\begin{array}{c}
n+q-3 \\
q-1
\end{array}\right)-\left(\begin{array}{c}
n+j-3 \\
j-1
\end{array}\right)\right\}=q(n-2)\left(\begin{array}{c}
n+q-3 \\
q-2
\end{array}\right)
$$

terms that must be determined. So $A\left(\phi \mid \phi_{n} ; \phi / \phi_{n-1}\right)$ is free abelian of rank $q(n-2)\left(\begin{array}{c}n+q-3 \\ q-2\end{array}\right)$. This was also proved by Bachmuth [2, Lemma 3].

If we now turn our attention to $A^{*}\left(\phi / \phi_{n} ; \phi / \phi_{n-1}\right), n \geqq 4$, condition (c) must hold so that not all the matrices included above are possible. By condition (b), $a_{q q}$ cannot involve the term $\sigma_{q}^{n-2}$, so the fact that the trace is 0 implies that whether or not $a_{q-1, q-1}$ involves $\sigma_{q}^{n-2}$ is already determined by the first $q-2$ diagonal terms. Thus one degree of freedom is destroyed. Also, by condition (c), $a_{a q}$ is determined by the other diagonal terms. This places certain constraints on the terms of the last row. Since there are $\left(\begin{array}{c}n+q-3 \\ a-1\end{array}\right)-1$ possible terms involved in $a_{a q},\left(\begin{array}{c}n+q-3 \\ a-1\end{array}\right)-1$ additional degrees of freedom are destroyed. Therefore, $A^{*}\left(\phi \mid \phi_{n} ; \phi / \phi_{n-1}\right)$ is free abelian of rank $q(n-2)\left(\begin{array}{c}n+q-3 \\ q-2\end{array}\right)-\left(\begin{array}{c}n+q-3 \\ q-1\end{array}\right)$. This again agrees with a result of Bachmuth [2, Theorem 2].

III. The main result. The main result of this paper is the following theorem.

THEOREM. If $\phi$ is free metabelian of rank $q \geqq 4$, then

$$
A^{*}\left(\phi / \phi_{n+2} ; \phi / \phi_{n+1}\right) \in K_{n}^{*},
$$

for every positive integer $n$.

This theorem gives rise to the following corollaries.

Corollary 1 . $A^{*}\left(\phi / \phi_{n} ; \phi / \phi_{2}\right) \in K^{*}$, for all $n \geqq 3$.

COROLlary 2. If $\phi$ is free metabelian of rank $q \geqq 4$, then, for every positive integer $n$, every automorphism of $\phi / \phi_{n}$ which is induced by an automorphism of $\phi$ is induced by an automorphism of the free group $F$ of rank $q$.

Corollary 1 follows from the theorem since if $\alpha \in A^{*}\left(\phi / \phi_{n} ; \phi / \phi_{2}\right)$ then $\alpha$ can be expressed in the form $\alpha=\alpha_{n-2} \cdots \alpha_{2} \alpha_{1}$, where

$$
\alpha_{i} \in A^{*}\left(\phi / \phi_{i+2} ; \phi / \phi_{i+1}\right) \text {. }
$$

Corollary 2 follows from Corollary 1 and the fact that $\phi / \phi_{2} \cong F / F_{2}$.

To prove the theorem, let us consider certain types of matrices $M=$ $I+\left(a_{i j}\right)$, where $I$ is the unit matrix. 
Type A. $a_{i j}=0$ for all $i, j$ except $i=r, j=s, t$, where $r \neq s, r \neq t$, $s<t ; a_{r s}$ is a monomial which involves $\sigma_{t}$ but no higher indexed $\sigma$ except possibly $\sigma_{r} ; a_{r t}=\left(-a_{r s} \sigma_{s}\right) / \sigma_{t}$.

Type B. $\quad a_{i j}=0$ for all $i, j$ except $i=r, s, j=r, s ; a_{r r}=\sigma_{r}^{\alpha} \sigma_{s}^{\beta}, \alpha \geqq 1$, $\beta \geqq 1 ; a_{s s}=-a_{r r} ; \dot{a}_{r s}=-\sigma_{r}^{\alpha+1} \sigma_{r}^{\beta-1} ; a_{s r}=\sigma_{r}^{\alpha-1} \sigma_{s}^{\beta+1}$.

Type C. $\quad a_{i j}=0$ for all $i, j$ except $i=r, s, j=r, s, t ; a_{r r}$ is a monomial involving $\sigma_{t} ; a_{s s}=-a_{r r} ; a_{r s}=a_{r r} ; a_{s r}=a_{s s} ; a_{r t}=-a_{r r}\left(\sigma_{r}+\sigma_{s}\right) / \sigma_{t}$; $a_{s t}=-a_{r t}$.

Type D. $a_{i j}=0$ for all $i, j$ except $i=r, j=r, s ; a_{r r}= \pm \sigma_{s} ; a_{r s}=\sigma_{r}$.

If $M=I+\left(a_{i j}\right)$, and $N=I+\left(b_{i j}\right)$ represent elements $\alpha$ and $\beta$ in $A^{*}\left(\phi / \phi_{n} ; \phi / \phi_{n-1}\right)$, then $I+\left(a_{i j}\right)+\left(b_{i j}\right)$ represents $\alpha \beta$. Therefore, to prove the theorem, it is sufficient to prove the following lemma:

LEMMA. For each positive integer $n$, every possible matrix of types $\mathrm{A}, \mathrm{B}, \mathrm{C}$, or $\mathrm{D}$, with terms of degree $n$ is in $K_{n}^{*}$.

(Note that there are no matrices of Type B if $n=1$, and that if $n>1$ there are no matrices of Type D.)

The theorem follows easily from the lemma, since any matrix in $A^{*}\left(\phi / \phi_{n+2} ; \phi / \phi_{n+1}\right)$ can be built up by first using matrices of Types $\mathrm{B}, \mathrm{C}$, and $\mathrm{D}$ to give us the diagonal terms and then using matrices of Type $\mathrm{A}$ to fix up the nondiagonal terms starting with the first column and progressively working our way to the last.

For example, the matrix

$$
\left(\begin{array}{cccc}
1+\sigma_{1} \sigma_{2}^{2}-\sigma_{3}^{3} & -\sigma_{1}^{2} \sigma_{2} & \sigma_{1} \sigma_{3}^{2} & 0 \\
\sigma_{2}^{3}+\sigma_{1} \sigma_{3} \sigma_{4} & 1-\sigma_{1} \sigma_{2}^{2} & -2 \sigma_{1}^{2} \sigma_{4} & \sigma_{1}^{2} \sigma_{3} \\
0 & 0 & 1 & 0 \\
\sigma_{3}^{3} & 0 & -\sigma_{3}^{2} \sigma_{4}-\sigma_{1} \sigma_{3}^{2} & 1+\sigma_{3}^{3}
\end{array}\right)
$$

is equal to the product of the matrices

$$
\begin{gathered}
\left(\begin{array}{cccc}
1+\sigma_{1} \sigma_{2}^{2} & -\sigma_{1}^{2} \sigma_{2} & 0 & 0 \\
\sigma_{2}^{3} & 1-\sigma_{1} \sigma_{2}^{2} & 0 & 0 \\
0 & 0 & 1 & 0 \\
0 & 0 & 0 & 1
\end{array}\right), \\
\left(\begin{array}{cccc}
1-\sigma_{3}^{3} & 0 & \sigma_{3}^{2}\left(\sigma_{1}+\sigma_{4}\right) & -\sigma_{3}^{3} \\
0 & 1 & 0 & 0 \\
0 & 0 & 1 & 0 \\
\sigma_{3}^{3} & 0 & -\sigma_{3}^{2}\left(\sigma_{1}+\sigma_{4}\right) & 1+\sigma_{3}^{3}
\end{array}\right),
\end{gathered}
$$




$$
\left(\begin{array}{cccc}
1 & 0 & -\sigma_{3}^{2} \sigma_{4} & \sigma_{3}^{2} \\
0 & 1 & 0 & 0 \\
0 & 0 & 1 & 0 \\
0 & 0 & 0 & 1
\end{array}\right), \quad\left(\begin{array}{cccc}
1 & 0 & 0 & 0 \\
\sigma_{1} \sigma_{3} \sigma_{4} & 1 & 0 & -\sigma_{1}^{2} \sigma_{3} \\
0 & 0 & 1 & 0 \\
0 & 0 & 0 & 1
\end{array}\right)
$$

and

$$
\left(\begin{array}{cccc}
1 & 0 & 0 & 0 \\
0 & 1 & -\sigma_{1}^{2} \sigma_{4} & \sigma_{1}^{2} \sigma_{3} \\
0 & 0 & 1 & 0 \\
0 & 0 & 0 & 1
\end{array}\right)^{2}
$$

if we work mod $J^{4}$, which is permissible if we consider these matrices as elements of $A^{*}\left(\phi / \phi_{5} ; \phi / \phi_{4}\right)$.

PROOF OF THE LEMMA. We will proceed by induction on $n$.

For $n=1,\left\{L_{r s t}^{\epsilon}\right\}$ takes care of all matrices of Type A, $\left\{K_{r s}^{\epsilon}\right\}$ takes care of all matrices of Type D, and $\left\{\left(K_{r t}^{-1} L_{r t s}^{-1} L_{s r t}^{-1} K_{s t}\right)^{\epsilon}\right\}$ takes care of all matrices of Type C. Thus any matrix in $A^{*}\left(\phi / \phi_{3} ; \phi / \phi_{2}\right)$ is in $K_{1}^{*}=K^{*}$.

(Note that the only case in which any reduction mod $J^{2}$ has to be done is for matrices of Type $\mathrm{D}$ in which the diagonal term is $1+\sigma_{s}$, in which case $K_{r s}^{-1}$ must be reduced mod $J^{2}$. However, such a matrix cannot itself represent an automorphism of $\phi$, since its determinant is not a unit. Therefore, all matrices of the above types which do represent IA automorphisms of $\phi$ are in $K^{*}$.)

Suppose the lemma is true for $n \leqq k-1$. We want to prove it is true for $n=k$.

Let us begin with any matrix $M$ of Type A in which $r=1, s=2$, $t=q$, and with $a_{12}$ of degree $k$. There are two cases to consider.

Case 1. The largest indexed $\sigma$ which divides $a_{1 q}$ also divides $a_{12}$, but is not $\sigma_{1}$. (Suppose the $\sigma$ in question is $\sigma_{p} ; p$ may equal $q$, but $p \neq 1$.)

Let $N=I+\left(b_{i j}\right)$, where $b_{12}=\left(-a_{12}\right) / \sigma_{p}, b_{1 q}=\left(-a_{1 q}\right) / \sigma_{p}$, and $b_{i j}=0$ otherwise. Then $N$ is of Type A and degree $k-1$, so by the induction hypothesis $N \in K_{k-1}^{*}$. But it is easily verified that $M=K_{1 p} N K_{1 p}^{-1} N^{-1}$; so $M \in K_{k}^{*}$.

Case 2. The largest indexed $\sigma$ which divides $a_{1 q}$ is either $\sigma_{1}$ or else does not divide $a_{12}$.

But since $a_{12} \sigma_{2}=-a_{1 q} \sigma_{q}$, and since $k>1$, this can only happen if $a_{12}=\sigma_{1}^{k-1} \sigma_{q}$. Let $N=I+\left(b_{i j}\right)$, where $b_{32}=\sigma_{1}^{k-2} \sigma_{q}, b_{3 q}=-\sigma_{1}^{k-2} \sigma_{2}$, and $b_{i j}=0$ otherwise. Since $q \neq 3, N$ is again of Type A and degree $k-1$, and hence by induction is in $K_{k-1}^{*}$. But $M=K_{13} N K_{13}^{-1} N^{-1}$, so $M \in K_{k}^{*}$. (Note that we need the fact that $q>3$ to say that $N \in K_{k-1}^{*}$. This is not 
surprising, as, for $q=3$, the matrix

$$
\left(\begin{array}{ccc}
1 & \sigma_{1}^{2} \sigma_{3} & -\sigma_{1}^{2} \sigma_{2} \\
0 & 1 & 0 \\
0 & 0 & 1
\end{array}\right)
$$

which corresponds to the automorphism $\mu$ mentioned in the introduction, is not in $K^{*}$ [3, Theorem 2]. The above proof shows, however, that if $q \geqq 4$, the analog of $\mu$ is in $K^{*}$. Namely, if

$$
\begin{aligned}
\mu^{\prime}: x_{1} & \rightarrow x_{1}\left(x_{1},\left(\left(x_{2}^{-1}, x_{3}^{-1}\right), x_{1}^{-1}\right)\right) \\
& x_{j} \rightarrow x_{j},
\end{aligned}
$$

then $\mu^{\prime}$ is the automorphism of $\phi$ induced by

$$
\left.K_{1 q} L_{q 32} K_{q 1} L_{q 32}^{-1} K_{q 1}^{-1} K_{1 q}^{-1} K_{q 1} L_{q 32} K_{q 1}^{-1} L_{q 32}^{-1} .\right)
$$

So far we have shown that certain matrices of Type A and degree $k$ are in $K_{k}^{*}$. But all other matrices of Type A and degree $k$ can be obtained from these by performing the proper permutation on the subscripts, rows, and columns. Suppose $M^{\prime}$ can be obtained from $M$ by performing the permutation $\pi$ on the subscripts, rows, and columns of $M$; and suppose that $M$ is equal to some word $w\left(K_{i j}, L_{i j k}\right)$ in the $K_{i j}$ and $L_{i j k}$. Then

$$
M^{\prime}=w\left(K_{\pi(i), \pi(j)}, L_{\pi(i), \pi(j), \pi(k)}\right) .
$$

Therefore, all matrices of Type A and degree $k$ are in $K_{k}^{*}$.

In considering matrices $M$ of Type $\mathrm{B}$, let us begin with one for which $r=1, s=q$. Recall that $a_{11}=\sigma_{1}^{\alpha} \sigma_{q}^{\beta}, a_{1 q}=-\sigma_{1}^{\alpha+1} \sigma_{q}^{\beta-1}, a_{q q}=-\sigma_{1}^{\alpha} \sigma_{q}^{\beta}$, and $a_{q 1}=\sigma_{1}^{\alpha-1} \sigma_{q}^{\beta+1}$ are the only nonzero terms, and that $\alpha \geqq 1, \beta \geqq 1$. Again we consider cases.

Case 1. $\beta \geqq 2$. In this case, let $N=I+\left(b_{i j}\right)$, where $b_{11}=\sigma_{1}^{\alpha} \sigma_{q}^{\beta-1}$, $b_{1 q}=-\sigma_{1}^{\alpha+1} \sigma_{q}^{\beta-2}, b_{q 1}=\sigma_{1}^{\alpha-1} \sigma_{q}^{\beta}, b_{q q}=-\sigma_{1}^{\alpha} \sigma_{q}^{\beta-1}$, and all other $b_{i j}$ are zero. $N$ is a matrix of Type B and degree $k-1$, and so by induction $N \in K_{k-1}^{*}$. But $M=N K_{1 q}^{-1} N^{-1} K_{1 q}$, so $M \in K_{k}^{*}$.

Case 2. $\beta=1, \alpha \geqq 2$. This case is analogous to Case 1 , with $N$ chosen so that $b_{11}=\sigma_{1}^{\alpha-1} \sigma_{q}^{\beta}, b_{1 q}=-\sigma_{1}^{\alpha} \sigma_{q}^{\beta-1}, b_{q q}=-\sigma_{1}^{\alpha-1} \sigma_{q}^{\beta}$, and $b_{q 1}=\sigma_{1}^{\alpha-2} \sigma_{q}^{\beta+1}$. Again $N \in K_{k-1}^{*}$ and $M=N K_{q 1}^{-1} N^{-1} K_{q 1}$, so $M \in K_{k}^{*}$.

Case 3. $\alpha=\beta=1$. In this case, $k=2$ and $M=K_{1 q}^{-1} K_{q 1}^{-1} K_{1 q} K_{q 1} \in K_{2}^{*}$. Again we have only dealt with certain matrices of Type B, but all others can be handled by applying the proper permutations, so matrices of Type B and degree $k$ are in $K_{k}^{*}$.

(Note that in dealing with matrices of Types A and B of degree $k$, we 
have not had to resort to reduction $\bmod J^{k+1}$. Note also that matrices of Types $\mathrm{A}$ and $\mathrm{B}$ have determinant 1 and hence represent automorphisms of $\phi$. Thus we have shown that all automorphisms of $\phi$ which are of Type A or B are in $K^{*}$.)

Since for $k>1$ there are no matrices of Type D, we are left with considering matrices of Type C. Again we will start with a special matrix $M$, with $r=1, s=q$, and $t=2$.

Case 1. $a_{11}=x \sigma_{2}$, where $x$ is divisible by $\sigma_{p}$ for some $p \neq 1, q$. Choose the largest such $p$. Let $N=I+\left(b_{i j}\right)$, where $b_{q 1}=-x, b_{q p}=$ $x \sigma_{1} / \sigma_{p}$, and all other $b_{i j}$ are zero. Then $N$ is of Type $\mathrm{A}$, and by induction $N \in K_{k-1}^{*}$. Let $M^{\prime}=L_{12 q} N L_{12 q}^{-1} N^{-1}$. Then $M^{\prime} \in K_{k}^{*} . M$ and $M^{\prime}$ have the same diagonal terms of degree $k$. Therefore, multiplying $M^{\prime}$ by the proper matrices of degree $k$ and Type A and reducing mod $J^{k+1}$ will give $M$. But since matrices of degree $k$ and Type A have already been shown to be in $K_{k}^{*}, M \in K_{k}^{*}$.

Case 2. $a_{11}=\sigma_{1}^{\alpha} \sigma_{2} \sigma_{q}^{\beta}, \alpha+\beta=k-1, \alpha \geqq 1$. Let $N=I+\left(b_{i j}\right)$, where $b_{q 1}=\sigma_{1}^{\alpha-1} \sigma_{2} \sigma_{q}^{\beta}, b_{q 2}=-\sigma_{1}^{\alpha} \sigma_{q}^{\beta}$, and all other $b_{i j}$ are zero. Then $N$ is of Type A and degree $k-1$, so $N \in K_{k-1}^{*}$. Let $M^{\prime}=K_{1 q} N K_{1 q}^{-1} N^{-1}$. Then $M^{\prime} \in K_{k}^{*}$, and, as in Case $1, M$ and $M^{\prime}$ have the same diagonal terms of degree $k$. Therefore, multiplying $M^{\prime}$ by matrices of Type A and degree $k$, and reducing $\bmod J^{k+1}$, we find that $M \in K_{k}^{*}$.

Case 3. $a_{11}=\sigma_{2} \sigma_{q}^{k-1}, k \geqq 2$. Let $N=I+\left(b_{i j}\right)$, where $b_{12}=-\sigma_{q}^{k-1}$, $b_{1 q}=\sigma_{2} \sigma_{q}^{k-2}$, and all other $\bar{b}_{i j}$ are zero. Again, by induction, $N \in K_{k-1}^{*}$, and so $M^{\prime}=K_{q 1} N K_{q 1}^{-1} N^{-1} \in K_{k}^{*}$. But, as in Cases 1 and 2, this implies that $M \in K_{k}^{*}$.

Applying the proper permutations will now give us all matrices of Type $\mathrm{C}$, and hence the lemma and the theorem are proven.

(Note that in dealing with Type $\mathrm{C}$ matrices, we had to resort to reducing $\bmod J^{k+1}$. This means that if we consider matrices of Type $\mathrm{C}$ and degree $k$ as being automorphisms of $\phi$ (which we can do since the determinant is 1) rather than of $\phi / \phi_{k+2}$, then we cannot immediately conclude that they are in $K^{*}$. Since the matrices of Types A, B, C, and D are in some sense building blocks, a first step towards proving that $A\left(\phi ; \phi / \phi^{\prime}\right)=K^{*}$ might be to show that all matrices of Types A, B, C, and D which represent automorphisms of $\phi$ are in $K^{*}$. While we have shown that this is true for matrices of Types A, B, and D, the discussion above makes us skeptical about whether it is true for matrices of Type C. In particular, we have been unable to discover whether the matrix of Type $\mathrm{C}$ and degree 2 given by $r=1, s=q, t=2$, and $a_{11}=\sigma_{1} \sigma_{2}$ is in $K^{*}$. We conjecture that it is not, and that therefore $A\left(\phi ; \phi / \phi^{\prime}\right) \neq K^{*}$, even for $q \geqq 4$. If this conjecture turns out to be correct, then we would also conjecture that $A\left(\phi ; \phi / \phi^{\prime}\right)$ is in fact not finitely generated.) 


\section{REFERENCES}

1. S. Bachmuth, Automorphisms of free metabelian groups, Trans. Amer. Math. Soc. 118 (1965), 93-104. MR 31 \#4831.

2. - Induced automorphisms of free groups and free metabelian groups, Trans. Amer. Math. Soc. 122 (1966), 1-17. MR 32 \#7626.

3. O. Chein, IA automorphisms of free and free metabelian groups, Comm. Pure Appl. Math. 21 (1968), 605-629. MR 39 \#1537.

4. W. Magnus, Über n-dimensionale Gittertransformationen, Acta Math. 64 (1935), 353-367.

5. J. Nielsen, Die Gruppe der dreidimensionalen Gittertransformationen, Danske Vid. Selsk. Mat.-Fys. Medd. 12 (1924), 1-29.

Department of Mathematics, Temple University, Philadelphia, Pennsylvania 19122 